\title{
On Tamir's algorithm for solving the nonlinear complementarity problem
}

\author{
Uwe Schäfer ${ }^{1, *}$ \\ ${ }^{1}$ Institut für Angewandte und Numerische Mathematik, Universität Karlsruhe, D-76128 Karlsruhe, Germany.
}

Some comments concerning Tamir's algorithm for solving the nonlinear complementarity problem are given.

(C) 2007 WILEY-VCH Verlag GmbH \& Co. KGaA, Weinheim

\section{Introduction}

Given a vector $f=\left(f_{1}, \ldots, f_{n}\right)^{\mathrm{T}}$ of $n$ real, nonlinear functions of a real vector $x=\left(x_{1}, \ldots, x_{n}\right)^{\mathrm{T}}$, the nonlinear complementarity problem $N C P(f)$ is to find a vector $x$ such that

$$
f(x) \geq 0, \quad x \geq 0, \quad x^{\mathrm{T}} f(x)=0,
$$

or to show that no such vector exists (see Facchinei and Pang [2] or Harker and Pang [4]). Here, the $\geq$-sign is meant componentwise.

In 1974, Tamir [7] published an algorithm for solving the $N C P(f)$ for the case that $f$ is a so-called Z-function, where $f$ is called a Z-function if for any $x \in \mathbb{R}^{n}$ the functions $\varphi_{i j}(t):=f_{i}\left(x+t e_{j}\right), i \neq j, i, j=1, \ldots, n$ are antitone and $e_{j}$ denotes the $j$ th unit vector. Tamir's algorithm is a generalization of Chandrasekaran's algorithm which solves the linear complementarity problem for the case that the given matrix $M$ is a so-called Z-matrix (see Chandrasekaran [1]).

\section{Tamir's algorithm}

Tamir's algorithm is given in Table 1 , where $\mathbb{R}_{+}^{k}$ denotes the positive orthant of $\mathbb{R}^{k}$; i.e., $\mathbb{R}_{+}^{k}=\left\{x \in \mathbb{R}^{k}: x_{j} \geq 0, j=\right.$ $1, \ldots, k\}$. We remark that the pseudocode in Table 1 is not the original pseudocode presented by Tamir. We have removed the modified Jacobi process. Instead, we use the lines 5-7.

$$
\begin{aligned}
& \text { begin } \\
& k:=0 ; z:=0 ; J:=\emptyset \text {; } \\
& \text { if } f(z) \geq 0 \text { then goto } 10 \\
& \text { else repeat } k:=k+1 \text {; } \\
& \text { choose } i_{k} \in\{1, \ldots, n\} \text { with } f_{i_{k}}(z)<0 \text {; } \\
& J:=J \cup\left\{i_{k}\right\} \text {; } \\
& \text { let } J=\left\{i_{1}, \ldots, i_{k}\right\} \text { and } g^{(k)}: \mathbb{R}_{+}^{k} \rightarrow \mathbb{R}^{k} \text { be defined as } \\
& \left(\begin{array}{c}
t_{1} \\
\vdots \\
t_{k}
\end{array}\right) \mapsto\left(\begin{array}{c}
f_{i_{1}}\left(\sum_{j=1}^{k} t_{j} e_{i_{j}}\right) \\
\vdots \\
f_{i_{k}}\left(\sum_{j=1}^{k} t_{j} e_{i_{j}}\right)
\end{array}\right) \\
& \text { 5: } \quad \text { let } M^{(k)}:=\left\{t \in \mathbb{R}_{+}^{k}: g^{(k)}(t)=0, t_{j} \geq z_{i_{j}}, j=1, \ldots, k-1\right\} \text {; } \\
& \text { 6: } \quad \text { if } M^{(k)} \neq \emptyset \text { then } \\
& \text { 7: } \operatorname{begin} t^{(k)}:=\inf M^{(k)} ; z:=\sum_{j=1}^{k} t_{j}^{(k)} e_{i_{j}} \text { end } \\
& \text { else begin write(' } \mathrm{NCP}(f) \text { has no solution'); goto } 20 \text { end; } \\
& \text { until } f(z) \geq 0 \text {; } \\
& \text { 10: write('The solution is ', } z \text { ); } \\
& \text { 20: end. }
\end{aligned}
$$

Table 1 Tamir's algorithm

\footnotetext{
* Corresponding author E-mail: Uwe.Schaefer@math.uni-karlsruhe.de, Phone: +49 7216087746 , Fax: +49721 6083767
} 


\begin{tabular}{|c|c|c|}
\hline$n$ & $\tilde{s}$ & running time \\
\hline 10 & 1.349931 & $0.001 \mathrm{~s}$ \\
50 & 1.372619 & $0.017 \mathrm{~s}$ \\
100 & 1.379208 & $0.114 \mathrm{~s}$ \\
150 & 1.390799 & $0.720 \mathrm{~s}$ \\
200 & 1.389587 & $1.507 \mathrm{~s}$ \\
250 & 1.388859 & $3.962 \mathrm{~s}$ \\
500 & 1.387397 & $20.478 \mathrm{~s}$ \\
\hline
\end{tabular}

$\varepsilon=10^{-5}$

\begin{tabular}{|c|c|c|}
\hline$n$ & $\tilde{s}$ & running time \\
\hline 10 & 1.349931 & $0.001 \mathrm{~s}$ \\
50 & 1.372619 & $0.028 \mathrm{~s}$ \\
100 & 1.393210 & $0.201 \mathrm{~s}$ \\
150 & 1.390799 & $0.831 \mathrm{~s}$ \\
200 & 1.389587 & $2.192 \mathrm{~s}$ \\
250 & 1.388859 & $4.577 \mathrm{~s}$ \\
500 & 1.393042 & $29.514 \mathrm{~s}$ \\
\hline
\end{tabular}

$\varepsilon=10^{-11}$

\section{Numerical examples}

We consider the ordinary free boundary problem:

$$
\left.\begin{array}{l}
\text { Find } s>0 \text { and } z(x):[0, \infty) \rightarrow \mathbb{R} \text { such that } \\
\begin{array}{c}
z^{\prime \prime}(x)=\sqrt{1+z(x)^{2}}, \text { for } x \in[0, s], \\
z(0)=1, \quad z^{\prime}(s)=0, \\
z(x)=0, \text { for } x \in[s, \infty) .
\end{array}
\end{array}\right\}
$$

One can show that (1) has a unique solution, say $\{\hat{s}, \hat{z}(x)\}$, and that $\hat{s} \leq \sqrt{2}$, see Schäfer [5] and Thompson [8]. Choosing $n \in \mathbb{N}$ and setting $l:=\frac{1}{n+1} \sqrt{2}, x_{i}:=i \cdot l, z_{i}: \approx \hat{z}\left(x_{i}\right), i:=1, \ldots, n$, the $N C P(f)$ is arising with $f(z)=M z+\Phi(z)+q$ where

$$
M=\left(\begin{array}{rrrrr}
2 & -1 & 0 & \cdots & 0 \\
-1 & 2 & -1 & \ddots & \vdots \\
0 & \ddots & \ddots & \ddots & 0 \\
\vdots & \ddots & -1 & 2 & -1 \\
0 & \cdots & 0 & -1 & 2
\end{array}\right), \Phi(z)=l^{2}\left(\begin{array}{c}
\sqrt{1+z_{1}^{2}} \\
\vdots \\
\vdots \\
\vdots \\
\sqrt{1+z_{n}^{2}}
\end{array}\right), q=\left(\begin{array}{r}
-1 \\
0 \\
\vdots \\
\vdots \\
0
\end{array}\right) .
$$

Obviously, $f$ is a continuous Z-function. Furthermore, it is well-known that $M$ is regular satisfying $M^{-1} \geq O$. Therefore, it is easy to see that $f(z), z \geq 0$ is injective. As a result, applying Tamir's algorithm for solving $N C P(f)$, all sets $M^{(k)}$ are either empty or a singleton. In contrast to the original paper of Tamir [7], the method for calculating a zero of $g^{(k)}$ is not fixed in Table 1. So, it is left to the programmer which method for calculating a zero is chosen.

The results presented in Table 2 are based on the following implementation (see Hammer [3]): The input data are $n$ and the tolerance $\varepsilon>0$. As the method for calculating a zero of $g^{(k)}$ Newton's method was chosen, where

$$
t_{\text {start }}:=\left\{\begin{array}{cc}
0 & \text { if } k=1 \\
\left(\begin{array}{c}
t^{(k-1)} \\
0
\end{array}\right) & \text { if } k>1
\end{array}\right.
$$

was taken as the starting point, respectively. If $z_{i}>0$ and $z_{i+1}=0$, then $\tilde{s}:=\frac{1}{2}\left(x_{i}+x_{i+1}\right)$ was taken as an approximation for $\hat{s}$. See Table 2 for some examples. Note, that the exact value of $\hat{s}$ satisfies $\hat{s} \in[1.393206,1.397715]$; see Schäfer [6].

\section{References}

[1] R. Chandrasekaran, A special case of the complementary pivot problem, Opsearch, 7, 263-268 (1970).

[2] F. Facchinei and J.-S. Pang, Finite-Dimensional Variational Inequality and Complementarity Problems, Volume I + II, Springer-Verlag (2003).

[3] D. Hammer, Eine Verallgemeinerung des Algorithmus von Chandrasekaran zur Lösung nichtlinearer Komplementaritätsprobleme, Diplomarbeit, Universität Karlsruhe (2006).

[4] P. T. Harker and J.-S. Pang, Finite-dimensional variational inequality and nonlinear complementarity problems: A survey of theory, algorithms and applications, Math. Programming, 48, 161-220 (1990).

[5] U. Schäfer, Unique solvability of an ordinary free boundary problem, Rocky Mountain J. Math., 34, 341-346 (2004).

[6] U. Schäfer, Accelerated enclosure methods for ordinary free boundary problems, Reliab. Comput., 9, 391-403 (2003).

[7] A. Tamir, Minimality and complementarity properties associated with Z-functions and M-functions, Math. Programming 7, 17-31 (1974).

[8] R. C. Thompson, A note on monotonicity properties of a free boundary problem for an ordinary differential equation, Rocky Mountain J. Math. 12, 735-739 (1982). 